\title{
On Intersections of the Hyperbolicity Domain With Straight Lines
}

\author{
Vladimir Petrov Kostov ${ }^{1}$ \\ ${ }^{1}$ Université de Nice, Laboratoire de Mathématiques, Parc Valrose, Alpes Maritimes, France \\ Correspondence: Vladimir Petrov Kostov, Université de Nice, Laboratoire de Mathématiques, Parc Valrose, Alpes \\ Maritimes, France. E-mail: Vladimir.KOSTOV@unice.fr
}

Received: March 22, 2014 Accepted: April 30, 2014 Online Published: June 26, 2014

doi:10.5539/jmr.v6n3p1 URL: http://dx.doi.org/10.5539/jmr.v6n3p1

\begin{abstract}
We consider the family of polynomials $x^{n}+a_{1} x^{n-1}+a_{2} x^{n-2}+\cdots+a_{n}, a_{i} \in \mathbf{R}$, and its hyperbolicity domain $\Pi_{n}$, i.e. the set of values of the coefficients $a_{i}$ for which the polynomial is with real roots only. We prove that for $0 \leq k \leq n-2$ there exist generic straight lines in $\mathbf{R}^{n} \cong O a_{1} \ldots a_{n}$ intersecting $\Pi_{n}$ along $k$ segments and two half-lines.
\end{abstract}

Keywords: hyperbolic polynomial, hyperbolicity domain

AMS Classification: 12D10

\section{Introduction}

\subsection{The Hyperbolicity Domain}

A real non-constant polynomial in one variable is (strictly) hyperbolic if it has only real (and distinct) roots. Consider the general family of monic real degree $n$ polynomials

$$
P(x, a):=x^{n}+a_{1} x^{n-1}+a_{2} x^{n-2}+\cdots+a_{n} .
$$

Its hyperbolicity domain $\Pi_{n} \subset \mathbf{R}^{n} \cong O a_{1} \ldots a_{n}$ is the set of values of the coefficients $a_{i}$ for which the polynomial is hyperbolic. In what follows we allow a slight abuse of terminology by identifying the points of the domain $\Pi_{n}$ with the corresponding hyperbolic polynomials. We set

$$
\Pi_{n, 0}:=\Pi_{n} \cap\left\{a_{1}=0\right\} \subset \mathbf{R}^{n-1} \cong O a_{2} \ldots a_{n} \text { and } \Pi_{n}^{*}:=\Pi_{n, 0} \cap\left\{a_{2}=-1\right\} .
$$

The set $\Pi_{n, 0}$ is of interest to us because a polynomial $P$ can be transformed by the shift $x \mapsto x-a_{1} / n$ into one with $a_{1}=0$. The sets $\Pi_{n}$ and $\Pi_{n, 0}$ are invariant under the one-parameter groups of quasi-homogeneous dilatations

$$
x \mapsto e^{\tau} x, a_{j} \mapsto e^{j \tau} a_{j}, \quad \tau \in \mathbf{R} .
$$

Therefore a hyperbolic polynomial $P$ with $a_{1}=0, a_{2} \neq 0$ can be transformed into one with $a_{1}=0, a_{2}=-1$ (If $a_{1}=0$ and $a_{2} \neq 0$, then $a_{2}<0$ because $a_{2}=-\left(x_{1}^{2}+\cdots+x_{n}^{2}\right) / 2$, where $x_{i}$ are the roots of $P$. The only hyperbolic polynomial with $a_{1}=a_{2}=0$ is $x^{n}$ ).

Definition 1 We use only the letters $x$ and $y$ to denote independent variables. We denote by $\Delta[P]$ the discriminant of the family of polynomials $P$, i.e. the set of values of the coefficients $a_{i}$ for which $P$ has a multiple root in $x$. A similar notation is used for the discriminants of all other polynomial families used in the paper. The discriminant $\Delta[P]$ is defined as the zero set of a polynomial in the coefficients $a_{i}$. It can be considered in the context of real or of complex coefficients.

In the present paper we are interested in pencils of real monic polynomials of the form $t Q+(1-t) R, t \in \mathbf{R}$, $\operatorname{deg} Q=\operatorname{deg} R=n$. We consider the question how many times can such a pencil intersect the hyperbolicity domain. A classical result (see Obrechkoff, 1963/2003) says that all polynomials of the pencil are strictly hyperbolic if and only if both $Q$ and $R$ are strictly hyperbolic and their roots interlace.

Remark 2 (1) The discriminant $\Delta[P]$ is defined as the set of zeros of the determinant $D$ of the Sylvester matrix $S\left(P, P^{\prime}\right)$ of the polynomials $P$ and $P^{\prime}$. The matrix is of size $(2 n-1) \times(2 n-1)$. Suppose that all coefficients $a_{i}$ are affine functions $\mu_{i} s+v_{i}$ of a (real or complex) parameter $s$. The first column of $S\left(P, P^{\prime}\right)$ does not depend on $s$ while 
in any of its other columns there are at least two entries which are affine functions of $s$. Therefore it is to be expected that typically $\operatorname{deg} D=2 n-2$. One can show that the coefficient of $s^{2 n-2}$ in $D$ is a non-trivial homogeneous degree $2 n-2$ polynomial in the coefficients $\mu_{i}$. Therefore a generic straight line intersects $\Delta[P]$ at $2 n-2$ points. In the case when all coefficients are real part (or none or all) of the points are real, the remaining ones form complex pairs. In the context of $\Pi_{n, 0}$ (i. e. if one sets $a_{1}=0$ ) one has $\operatorname{deg} D=2 n-3$ (because two columns of $S\left(P, P^{\prime}\right)$ are independent of $s$ ). In the same way for $a_{1}=0, a_{2}=-1$ (i.e. in the context of $\Pi_{n}^{*}$ ) one has $\operatorname{deg} D=2 n-4$. Hence in these cases a generic line intersects $\Delta[P]$ at $2 n-3$ and $2 n-4$ points respectively.

(2) For more details about the hyperbolicity domain see, for example, Kostov (1989, 2011), Méguerditchian (1991a, 1991b, 1992). An impetus to study its properties was given by the articles (Arnold, 1986) and (Givental, 1987). Two particular classes of hyperbolic polynomials and the corresponding domains are studied in Kostov (2004, 2005a, 2005b, 2007). See also Chebotarev (1942), Meiman $(1938,1940)$ and Meiman and Chebotarev (1934).

\subsection{Examples}

In the present subsection we show how the hyperbolicity domain looks like for small values of $n$. We discuss what can be the intersection of a straight line with the hyperbolicity domain.

(1) The domains $\Pi_{1}$ and $\Pi_{1,0}$ are just $\mathbf{R}^{1}$ and its origin.

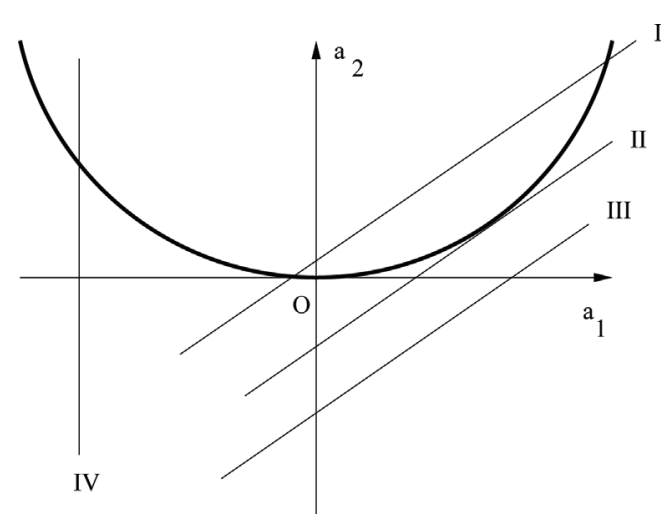

Figure 1 . The hyperbolicity domain for $n=2$ and the lines intersecting it

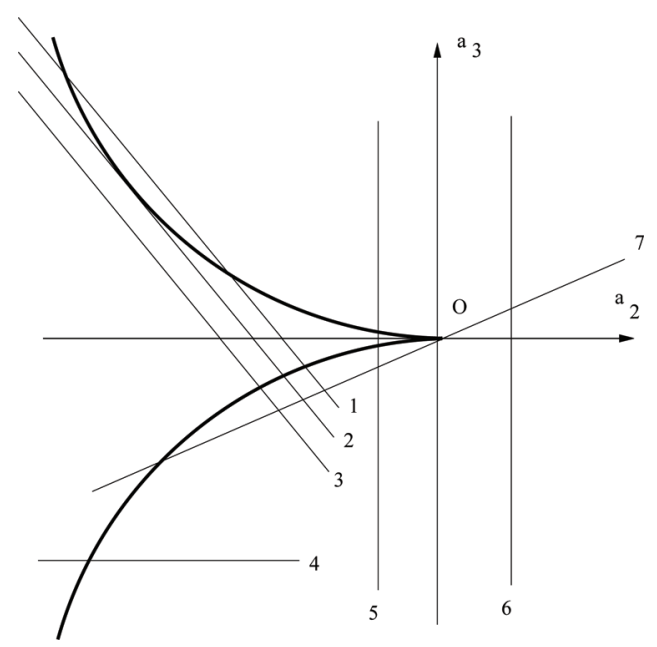

Figure 2. The hyperbolicity domain for $n=3, a_{1}=0$, and the lines intersecting it

(2) One has $\Pi_{2}=\left\{a_{1}^{2}-4 a_{2} \geq 0\right\}$, see Figure 1. A generic straight line in $\mathbf{R}^{2}$ either belongs to the interior of $\Pi_{2}$ (see line III) or intersects $\Pi_{2}$ along two half-lines (line I). A non-generic line is either tangent to the parabola $a_{1}^{2}=4 a_{2}$ (line II) or is parallel to the $a_{2}$-axis and hence intersects $\Pi_{2}$ along a half-line (line IV). The domain $\Pi_{2,0}$ is the half-line $\left\{a_{2} \leq 0\right\} \subset \mathbf{R}^{1} \cong O a_{2}$. 
(3) The domain $\Pi_{3,0}$ is the part of $\mathbf{R}^{2}$ delimited by the semi-cubic parabola $4 a_{2}^{3}+27 a_{3}^{2}=0$ and containing the half-line $a_{2} \leq 0$, see Figure 2. Any generic line is neither vertical nor horizontal hence intersects it along a halfline or a half-line and a segment, see lines 1 and 3 on Figure 2. Within the class of non-generic lines there is the subclass of "least non-generic" ones. It consists of the vertical lines intersecting the domain along segments (line 5), the vertical lines not intersecting it at all (line 6), the horizontal lines not passing through the origin (line 4, they intersect the domain along half-lines), the non-horizontal lines passing through the origin (line 7, they intersect the domain along a half-line and at the origin) and the lines tangent to the semi-cubic parabola, but not at the origin (line 2). Finally there are the "most non-generic" lines-the two coordinate axes.

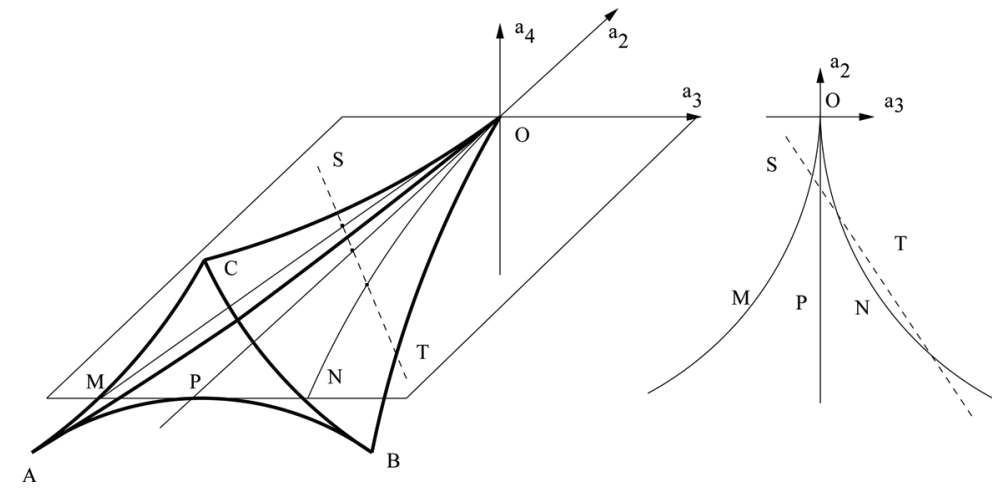

Figure 3. The hyperbolicity domain for $n=4, a_{1}=0$, and the lines intersecting it

(4) The domain $\Pi_{4,0}$ is shown on the left of Figure 3. This is the curvilinear pyramid $O A B C$ having a transversal self-intersection along the open arc $C O$ and semi-cubic singularities in the transversal cross-sections at the interior points of the open arcs $A O$ and $B O$. The concavity of the faces of the pyramid is as shown on Figure 3 . The intersection of $\Pi_{4,0}$ with the coordinate plane $O a_{2} a_{3}$ is the closure of the domain in $O a_{2} a_{3}$ delimited by the semicubic parabola $M O N$ and containing the negative $a_{2}$-half-axis (i.e. $O P$ ).

The right part of Figure 3 shows the $O a_{2} a_{3}$-plane and the semi-cubic parabola MON. A straight line $S T$ belonging to the $\mathrm{Oa}_{2} a_{3}$-plane and as shown on Figure 3 intersects $\Pi_{4,0}$ along a segment and a half-line. The segment contains inside itself a point at which the line $S T$ is tangent to the border of $\Pi_{4,0}$; this point is in fact $S T \cap O P$. Hence when the line $S T$ is shifted slightly while remaining parallel to itself so that the $a_{2}$ - and $a_{3}$-coordinates of its points remain the same while the $a_{4}$-coordinate decreases, the intersection $S T \cap \Pi_{4,0}$ will consist of two segments and a half-line.

We are not giving the complete description of the possible cases of intersection of a line with $\Pi_{4,0}$. However we prove the following proposition:

Proposition 3 Any straight line not intersecting $\Pi_{4,0}$ either belongs to a plane $\left\{a_{2}=\right.$ const $\}$ or its intersection with the half-space $\left\{a_{2} \leq 0\right\}$ belongs to the set $\left\{a_{2} \leq 0, a_{3}=0, a_{4}<0\right\}$.

Remark 4 Suppose that a straight line belongs to a plane $\left\{a_{2}=r\right\}$. If $r>0$, then any such straight line does not intersect $\Pi_{4,0}$ because $\Pi_{4,0} \subset\left\{a_{2} \leq 0\right\}$. If $r \leq 0$, then the line does not intersect $\Pi_{4,0}$ exactly when it avoids the compact set $\Pi_{4,0} \cap\left\{a_{2}=r\right\}$. This set is obtained from $\Pi_{4}^{*}$ by a linear transformation, see formula (1). Hence it is necessary and sufficient for the line to avoid the (rectilinear) triangle $A B C$, see Figure 3.

Proof of Proposition 3. If a line does not belong to a plane $\left\{a_{2}=\right.$ const $\}$, then it can be parametrized as follows:

$$
a_{2}=-s, a_{3}=\alpha s+\beta, a_{4}=\gamma s+\delta, \quad s \in \mathbf{R} .
$$

If $\gamma>0$ or $\gamma=0<\delta$, then the absolute values of the $a_{3}$ - and $a_{4}$-coordinates of a point of the line increase at most linearly in $s$. Consider a half-disk $\left\{a_{2}=-1, a_{3}^{2}+a_{4}^{2} \leq \varepsilon, a_{4} \geq 0\right\}$, where $\varepsilon>0$ is so small that the half-disk belongs to $\Pi_{4}^{*}$. Consider the image of the half-disk under the one-parameter group of diffeomorphisms defined by formula (1). This image belongs to $\Pi_{4,0}$. Set $s=e^{t}$. Then the $a_{3}$ - and $a_{4}$-coordinates of the image of each point of the interior of the half-disk increase respectively as $s^{3 / 2}$ and $s^{2}$. Hence for $s$ sufficiently large the image of the half-disk contains the point of the straight line.

For $\gamma=\delta=0$ and $\alpha \neq 0$ or $\alpha=0 \neq \beta$ the reasoning is much the same. For $s$ large enough the point of the line 
belongs to the border of the image of the half-disk, but is not at its centre (i.e. not on the $a_{2}$-axis), hence the point belongs to the interior of $\Pi_{4,0}$.

Suppose that $\gamma<0, \alpha \neq 0$ or $\gamma=0>\delta, \alpha \neq 0$. The set $\Pi_{4}^{*}$ contains a domain of the form $\left\{a_{2}=-1,\left|a_{3}\right| \leq \varepsilon\right.$, $\left.0 \geq a_{4} \geq-g a_{3}^{2}\right\}$ for some $\varepsilon>0$ and $g>0$. Indeed, the arc $A P B$ has an ordinary tangency with the line $M N$ (see Kostov, 2011). The image of this domain under the one-parameter group of diffeomorphisms (1) is the domain $\left\{a_{2}=-s,\left|a_{3}\right| \leq \varepsilon s^{3 / 2}, 0 \geq a_{4} \geq-s g a_{3}^{2}\right\}$. For $s>0$ sufficiently large this domain contains the corresponding point of the straight line, see (2).

For $\gamma<0, \alpha=0 \neq \beta$ or $\gamma=0>\delta, \alpha=0 \neq \beta$ the point of the line belongs to the border of the image of the domain, but is not on the $a_{2}$-axis, hence the point belongs to the interior of $\Pi_{4,0}$.

Hence the line might not intersect $\Pi_{4,0}$ only for $\alpha=\beta=0$, i.e. when the straight line belongs to the $O a_{2} a_{4}$-plane. The line must not intersect the set $\left\{a_{2} \leq 0, a_{3}=0, a_{4} \geq 0\right\}$. Indeed, in this case it will intersect $\Pi_{4,0}$ because it will either intersect $O P$ (the negative $a_{2}$-half-axis) or the arc $O C$ (the $a_{4}$-coordinate of a point of the arc $O C$ increases as $s^{2}$ while the $a_{4}$-coordinate of the point of the line increases at most linearly in $s$ ). Hence the intersection of the line with the half-space $\left\{a_{2} \leq 0\right\}$ belongs to the set $\left\{a_{2} \leq 0, a_{3}=0, a_{4}<0\right\}$.

\subsection{The Basic Result}

The main result of the paper is the following theorem:

Theorem 5 For $n \geq 3$ the following statements hold true:

(1) For $a_{1}=0$ a generic straight line in $\mathbf{R}^{n-1} \cong O a_{2} \ldots a_{n}$ intersects $\Pi_{n, 0}$ along $k$ segments and a half-line, where $0 \leq k \leq n-2$, and for any such $k$ there exists such a generic straight line.

(2) For $0 \leq k \leq n-2$ there exist generic straight lines in $\mathbf{R}^{n} \cong O a_{1} \ldots a_{n}$ intersecting $\Pi_{n}$ along $k$ segments and two half-lines.

To prove or disprove the following conjecture would also be of interest:

Conjecture 6 Any straight line in $\mathbf{R}^{n}$ which is not parallel to any of the coordinate hyperplanes intersects the interior of $\Pi_{n}$.

\section{Proof of Theorem 5}

For $n=3$ part (1) of the theorem can be checked directly, see part (3) of Subsection 1.2.

We prove part (1) by induction on $n$. Suppose that it is proved for $n-1$ and that $n \geq 4$. The domain $\Pi_{n}^{*}$ is compact (as $x_{1}^{2}+\cdots+x_{n}^{2}=2$, all roots $x_{k}$ and all coefficients $a_{i}$ are bounded). This domain contains the polynomial $U:=(x-b)^{n-1}(x+(n-1) b), b=\sqrt{2 / n(n-1)}$.

In a neighbourhood of the point $U \in \mathbf{R}^{n}$ (respectively $U \in \mathbf{R}^{n-1}$ ) one can present any polynomial in the form $(x-u) G(x, \lambda)$ (respectively $\left.\left(x-\lambda_{1}\right) G\right)$, where $G(x, \lambda):=x^{n-1}+\lambda_{1} x^{n-2}+\cdots+\lambda_{n-1}$ and $u$ and $\lambda_{1}$ are close to $-(n-1) b$.

Lemma 7 In a neighbourhood of the point $U \in \mathbf{R}^{n}$ (respectively $U \in \mathbf{R}^{n-1}$ ) one can choose as local coordinates the quantities $\left(\lambda_{1}, \lambda_{2}, \ldots, \lambda_{n-1}, u\right)$ (respectively $\left(\lambda_{1}, \lambda_{2}, \ldots, \lambda_{n-1}\right)$ ).

The lemma is proved in the next section.

Set $y:=(x-b) / v$, where $v>0$ is a parameter. Hence $v^{n-1} U=y^{n-1}(n b+v y)$. To choose $v>0$ small means to magnify the local picture around the point $U$.

Consider the family of polynomials in $y$

$$
v^{n-1} \tilde{U}:=(n b+v y) V \text {, where } V:=y^{n-1}+c_{2} y^{n-3}+\cdots+c_{n-1} .
$$

Consider the discriminant $\Delta\left[v^{n-1} \tilde{U}\right]$. Hence $\Delta\left[v^{n-1} \tilde{U}\right]$ can be regarded as a family of hypersurfaces in the space $O c_{2} \ldots c_{n-1}$ analytically depending on $v$.

By inductive assumption for any $0 \leq k \leq n-3$ there exist generic straight lines in $\mathbf{R}^{n-2} \cong O c_{2} \ldots c_{n-1}$ intersecting the hyperbolicity domain of the family of polynomials $V$ along $k$ segments and a half-line. Suppose that for any such $k$ such a generic line $L_{k}$ is fixed. Genericity of the lines implies that the intersections of these lines with the discriminant $\Delta[V]$ are transversal. Hence the intersections of these lines (when considered as relative to the family $v^{n-1} \tilde{U}$, not $V$ ) will be transversal for $v \geq 0$ small enough as well. 
For any $v>0$ small enough the straight line $L_{k}$ defines a straight line $M_{k}$ in $O a_{2} \ldots a_{n}$. Indeed, the coefficients $a_{i}$ are affine functions (depending on $v$ as on a parameter) of the coefficients $c_{i}$. One can introduce a parameter $w \in \mathbf{R}$ and express the coordinates of a point of the line $L_{k}$ in the form $c_{i}=p_{i, k} w+q_{i, k}, p_{i, k}, q_{i, k} \in \mathbf{R}, i=1, \ldots, n-1$. Thus the coordinates of a point of the line $M_{k}$ can also be expressed by affine functions (depending on $v$ ) of $w$.

The lines $L_{k}$ and the coordinates $c_{i}$ can be considered also as complex ones. Hence each line $L_{k}$ intersects transversally the complex discriminant $\Delta[V]$ at $2 n-5$ complex points out of which $2 k+1$ are real. Genericity implies that all these points are finite.

Fix $v$ small enough. The line $M_{k}$ intersects the domain $\Pi_{n}^{*}$ along $k$ segments whose endpoints are close to the point $U$ and along another segment only one of whose endpoints is close to $U$. It is true that the line $L_{k}$ intersects the hyperbolicity domain of the family of polynomials $V$ along a half-line, but the presentation of a polynomial in the form $(x-u) G(x, \lambda)$ (respectively $\left.\left(x-\lambda_{1}\right) G\right)$ is defined only locally, in a neighbourhood of the point $U$. It implies the existence of the endpoints of the $k$ segments and of the half-line as intersection points of the line $L_{k}$ with the discriminant $\Delta[V]$. However the line $M_{k}$ a priori intersects the compact domain $\Pi_{n}^{*}$ only along segments and/or points, not along a half-line. Hence its intersection with $\Pi_{n}^{*}$ consists of $k+1$ segments.

The line $M_{k}$ belongs to a hyperplane $\left\{a_{2}=\right.$ const $\}$. It has $2 n-4$ intersection points with $\Delta[V]$ out of which $2 n-5$ are close to the point $U$ and $2 k+2$ are real. Perturb the position of $M_{k}$ so that it no longer belongs to a hyperplane of the form $\left\{a_{2}=\right.$ const $\}$. Such a generic perturbation introduces a single "distant" point which is real (otherwise it would be a complex conjugate pair). This point is the endpoint of a half-line of the intersection of $M_{k}$ with $\Pi_{n, 0}$.

To finish the proof of the induction step for part (1) one should prove that there exist generic lines intersecting $\Pi_{n, 0}$ along half-lines and no segments. Consider a monic strictly hyperbolic polynomial $P$ with roots $x_{1}<x_{2}<\cdots<x_{n}$. Consider a polynomial $Q:=\left(x-z_{1}\right) \cdots\left(x-z_{n-2}\right)$, where $x_{i}<z_{i}<x_{i+1}, i=1, \ldots, n-2$. The interlacing of the roots implies that for any $t \in \mathbf{R}$ the polynomial $S_{t}:=P+t Q$ has at least $n-2$ real roots $\xi_{i}$ such that any interval $\left(x_{i}, x_{i+1}\right)$ and any interval $\left(z_{i}, z_{i+1}\right), i=1, \ldots, n-3$ contains exactly one of the roots $\xi_{i}$.

There exists $t_{0}$ such that the rightmost root of the polynomial $S_{t_{0}}$ is a double one. For $t>t_{0}, S_{t}$ has a complex conjugate pair of roots. Indeed, as $S_{t}=S_{t_{0}}+\left(t-t_{0}\right) Q$ and as $Q>0$ for $x>z_{n-2}$, the polynomial $S_{t}$ is positive for $x>z_{n-2}$. On the other hand it has at most $n-1$ and at least $n-2$ roots that are distinct and $\leq z_{n-2}$. For any $t \in \mathbf{R}$ the polynomial $S_{t}$ is of degree $n$. Hence for $t>t_{0}$ the polynomial $S_{t}$ has exactly $n-2$ real distinct roots.

For $t<t_{0}$ a similar reasoning shows that $S_{t}$ has two distinct real roots that are $>z_{n-2}$. As $S_{t}$ has at least $n-3$ distinct real roots that are $<z_{n-2}$, it has at least $n-1$ distinct real roots. Hence it has $n$ real roots (because $\operatorname{deg} S_{t}=n$ ). Its roots that are not greater than $z_{n-2}$ are distinct hence all its roots are distinct. This means that the straight line defined by the family of polynomials $S_{t}$ has the Property (A): it has a double root only for $t=t_{0}$, it has $n$ distinct real roots for $t<t_{0}$ and exactly $n-2$ distinct real roots for $t>t_{0}$.

The roots $z_{i}$ are required only to interlace with the roots $x_{1}, \ldots, x_{n-2}$. Hence any nearby line defines a family $S_{t}$ which satisfies the Property (A) for some $t^{*}$ close to $t_{0}$. This means that the straight line defined by the family $S_{t}$ is generic. This line belongs to $\mathrm{Oa}_{2} \ldots a_{n}$ because the coefficients of $x^{n-1}$ are 0 in $P$ and in $Q$.

Part (2) of the theorem follows from part (1). Indeed, suppose that a generic line $M_{k} \subset \Pi_{n, 0}$ as above is constructed $(-1 \leq k \leq n-3)$. Perturb this line so that it no longer belongs to a hyperplane of the form $\left\{a_{1}=\right.$ const $\}$. Such a generic perturbation introduces a single "distant" point which (not being a complex conjugate pair) is real. This point is the endpoint of a half-line of the intersection of $M_{k}$ with $\Pi_{n}$.

\section{Proof of Lemma 7}

The following formulas connect the quantities $a_{i}$ with the quantities $u$ and $\lambda_{i}$ :

$$
a_{1}=\lambda_{1}-u, \quad a_{2}=\lambda_{2}-u \lambda_{1}, \ldots a_{n-1}=\lambda_{n-1}-u \lambda_{n-2}, \quad a_{n}=-u \lambda_{n-1} .
$$

Hence the Jacobian matrix of this change of variables equals

$$
\left(\begin{array}{rrrrrr}
-1 & 1 & 0 & \cdots & 0 & 0 \\
-\lambda_{1} & -u & 1 & \cdots & 0 & 0 \\
-\lambda_{2} & 0 & -u & \cdots & 0 & 0 \\
\vdots & \vdots & \vdots & \ddots & \vdots & \vdots \\
-\lambda_{n-2} & 0 & 0 & \cdots & -u & 1 \\
-\lambda_{n-1} & 0 & 0 & \cdots & 0 & -u
\end{array}\right) .
$$


To find its determinant add the first row multiplied by $u$ to the second one, then the second row multiplied by $u$ to the third one, ... then the $(n-1)$-st row multiplied by $u$ to the $n$-th one. The matrix becomes

$$
\left(\begin{array}{rccccc}
-1 & 1 & 0 & \cdots & 0 & 0 \\
-\lambda_{1}-u & 0 & 1 & \cdots & 0 & 0 \\
-\lambda_{2}-\lambda_{1} u-u^{2} & 0 & 0 & \cdots & 0 & 0 \\
\vdots & \vdots & \vdots & \ddots & \vdots & \vdots \\
-\lambda_{n-2}-\lambda_{n-3} u-\cdots-\lambda_{1} u^{n-3}-u^{n-2} & 0 & 0 & \cdots & 0 & 1 \\
-\lambda_{n-1}-\lambda_{n-2} u-\lambda_{n-3} u^{2}-\cdots-\lambda_{1} u^{n-2}-u^{n-1} & 0 & 0 & \cdots & 0 & 0
\end{array}\right) .
$$

Its entry in position $(n, 1)$ equals $-G(u, \lambda)$, so its determinant is $(-1)^{n+1} G(u, \lambda)$. The root $u$ is not close to $b$, therefore $G(u, \lambda) \neq 0$.

When one sets $u=\lambda_{1}$, then formulas (4) become

$$
a_{2}=\lambda_{2}-\lambda_{1}^{2}, \quad a_{3}=\lambda_{3}-\lambda_{1} \lambda_{2}, \ldots a_{n-1}=\lambda_{n-1}-\lambda_{1} \lambda_{n-2}, \quad a_{n}=-\lambda_{1} \lambda_{n-1} .
$$

The Jacobian matrix equals

$$
\left(\begin{array}{rrrrrr}
-2 \lambda_{1} & 1 & 0 & \cdots & 0 & 0 \\
-\lambda_{2} & -\lambda_{1} & 1 & \cdots & 0 & 0 \\
-\lambda_{3} & 0 & -\lambda_{1} & \cdots & 0 & 0 \\
\vdots & \vdots & \vdots & \ddots & \vdots & \vdots \\
-\lambda_{n-2} & 0 & 0 & \cdots & -\lambda_{1} & 1 \\
-\lambda_{n-1} & 0 & 0 & \cdots & 0 & -\lambda_{1}
\end{array}\right)
$$

Add the $k$ th row multiplied by $\lambda_{1}$ to the $(k+1)$-st one for $k=1,2, \ldots n-2$. This makes disappear all terms $-\lambda_{1}$ on the diagonal, the entry in position $(n, 1)$ becomes $-G\left(\lambda_{1}, \lambda\right) \neq 0$ (because $\lambda_{1}$ is close to $-(n-1) b$, not to $b$ ); the determinant equals $\pm G\left(\lambda_{1}, \lambda\right)$.

\section{Acknowledgements}

The author is grateful to B. Z. Shapiro and G. Blekherman for the statement of the problem and the useful discussions of this text.

\section{References}

Arnold, V. I. (1986). Hyperbolic polynomials and Vandermonde mappings. Functional Analysis and Applications, 20(2), 52-53.

Chebotarev, N. G. (1942). On R-integrable polynomials. Doklady Akademii Nauk SSSR, XXXV(3), 67-71.

Givental, A. B. (1987). Moments of random variables and the equivariant Morse lemma (Russian). Uspekhi Matematicheskikh Nauk, 42(2), 221-222.

Kostov, V. P. (1989). On the geometric properties of Vandermonde's mapping and on the problem of moments. Proceedings of the Royal Society of Edinburgh, A 112, 203-211.

Kostov, V. P. (2004). Very hyperbolic and stably hyperbolic polynomials. Comptes Rendus de l'Académie des Sciences, Paris, Série I, 339, 157-162.

Kostov, V. P. (2005a). Very hyperbolic polynomials. Functional Analysis and Its Applications, 39(3), 229-232. http://dx.doi.org/10.1007/s10688-005-0042-4

Kostov, V. P. (2005b). Very hyperbolic polynomials in one variable. Proceedings of the Royal Society of Edinburgh, A $135(4), 833-844$.

Kostov, V. P. (2007). Stably hyperbolic polynomials. Advanced Studies in Pure Mathematics 46. Singularities in Geometry and Topology, 95-104.

Kostov, V. P. (2011). Topics on hyperbolic polynomials in one variable. Paris, France: SMF, Panoramas et Synthèses 33.

Méguerditchian, I. (1991a). Géométrie locale des polynômes hyperboliques. Comptes Rendus de l'Académie des Sciences, Paris, Série I, 312(11), 849-852. 
Méguerditchian, I. (1991b). Géométrie du discriminant réel et des polynômes hyperboliques (Unpublished doctoral dissertation). University of Rennes, France.

Méguerditchian, I. (1992). A theorem on the escape from the space of hyperbolic polynomials. Mathematische Zeitschrift, 211, 449-460. http://dx.doi.org/10.1007/BF02571438

Meiman (Meymann), N. N. (1938). Sur les polynômes R-prolongeables. Matematicheskiy Sbornik, 3(45), 591650.

Meiman (Meymann), N. N. (1940). On continuable polynomials. II. On R- continuable polynomials. Memorial volume dedicated to academician D. A. Grave (Russian), 117-165.

Meiman, N. N., \& Chebotarev, N. G. (1934). On R-continuable polynomials. Proceedings of the Second All-Union Mathematics Congress 2 (pp. 6-9), Moscow: Akademiya Nauk.

Obrechkoff, N. (1963/2003). Zeros of polynomials (I. Dimovski \& P. Rusev, Trans.). Sofia: The Marin Drinov Academic Publishing House.

\section{Copyrights}

Copyright for this article is retained by the author(s), with first publication rights granted to the journal.

This is an open-access article distributed under the terms and conditions of the Creative Commons Attribution license (http://creativecommons.org/licenses/by/3.0/). 